\title{
The persistent challenge of inequality in Australia's health
}

Robyn Godding

Deputy Editor

mja@mja.com.au

doi: 10.5694/mjal4.c1020 ustralia remains a country with significant health inequalities. The Australian Institute of Health and Welfare (AIHW) reports that although Australians have a life expectancy of 82 years - one of the highest among Organisation for Economic Co-operation and Development (OECD) countries - and the second lowest overall mortality of OECD countries, there are inequalities in age-standardised mortality rates among population groups (http:// www.aihw.gov.au/publication-detail/?id=60129548021). In 2009-2011, males had a mortality rate 1.5 times that of females; people living in remote and very remote areas had a rate 1.4 times that of people in major cities; people living in the lowest socioeconomic status (SES) areas had a rate 1.3 times that of those in areas with the highest SES; and the mortality rate for Indigenous Australians was almost twice that for nonIndigenous Australians. These differences remained much the same as 10 years earlier. Yet not all mortality differences were negative. For example, Asian-born Australian residents had a mortality rate $36 \%$ lower than Australian-born residents.

The AIHW estimates that if all Australians had the same death rates as those in the highest SES group, there would have been 6013 fewer deaths from lung cancer - Australia's leading cause of cancer deaths — in 2009-2011. If Australians living in regional and remote areas had the same death rates as those living in urban areas, there would have been 825 fewer deaths from prostate cancer in the same period. That is, nearly 7000 excess deaths due to these two cancers in this 3-year period could be predicted and attributed to differences in area of residence and SES.

In this issue of the $M J A$, a series of oncology-related articles report inequalities that fit with those reported by the AIHW.

In their perspective article, Fox and Boyce (page 445) highlight how a recent Australia-wide study found that the $7 \%$ higher mortality for those living in rural areas compared with those living in urban areas had remained unchanged during the decade from 2001 to 2010. They also cite research which found that mortality was 45\% higher for Indigenous people with cancer than for non-Indigenous people.

A further example of cancer-related inequality for Indigenous Australians is provided by Parker and colleagues (page 470), who report that the incidence of hepatocellular carcinoma in the Northern Territory is 5.9 times higher in Indigenous people than in nonIndigenous people.

In relation to non-small cell lung cancer (NSCLC) in New South Wales, Currow and colleagues (page 475 ) calculated that if resection rates increased, the proportion of people with localised NSCLC dying in the 5 years after diagnosis would decrease by $10 \%$. They found that the resection rate was negatively associated with local health district of residence (with lower rates in remote and very remote areas), being older, having lower SES and not having private health insurance.

Hocking and colleagues (page 462) found that in South Australia, where there is a centralised model of cancer treatment, there was no difference in survival between people with metastatic colorectal cancer living in rural areas and those living in urban areas. This finding supports the AIHW estimates of deaths that might be avoided if people in rural areas had the same death rates as those in urban areas.

A positive development in relation to equity in cancer services is that, as reported by Grogan and Olver (page 435), the National Bowel Cancer Screening Program will be fully implemented by 2020. This means that all Australians aged 50-74 years will be invited to participate in biennial faecal occult blood test screening; currently, only subgroups of the eligible population are invited to participate. Modelling done by Cenin and colleagues (page 456) shows that, compared with not fully implementing the program until 2035, full implementation by 2020 has the potential to prevent an additional 35000 deaths from bowel cancer over the next 40 years.

\section{Cate Swannell
Careers Editor cswannell@mja.com.au}

Careers follows $p 494$

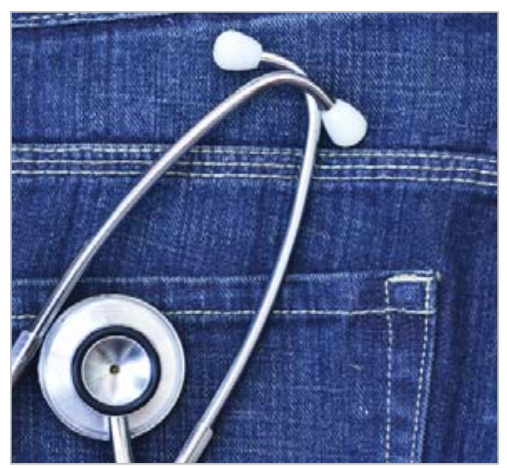

\section{General practice highs and lows}

Dr Ian Kamerman loves his job, both as a country GP and as president of the Rural Doctors Association of Australia. The Tamworth (NSW) GP talks about why the country life is the best of general practice worlds, and how the gap in access between rural and city practices can be narrowed (page C1). Dr Liz Marles is ready to get back to full-time practice after her 2-year term as president of the Royal Australian College of General Practitioners. It's been a rollercoaster ride, but one she's enjoyed (page C3). 